\title{
FINITE-DIMENSIONAL CHU SPACE, FUZZY SPACE AND THE GAME INVARIANCE THEOREM
}

\author{
NGUYEN NHUY, VU THI HONG THANH
}

\begin{abstract}
By constructing the notion " $(n+1)$-fuzzy functor", it is shown that the $(n+1)$-fuzzy category introduced in [3] is an equivalent system. Moreover, the game invariance theorem is proved in this note.

Tóm tăt. Chúng tôi đưa ra một lớp các hàm tử hiệp biển, được gọi là "(n+1)- hàm tư fuzzy", từ phạm trù các $n$ - tập hợp vào phạm trù các $(n+1)$ - không gian fuzzy; chl ra rằng $(n+1)$ - phạm trù fuzzy là một hệ thống tương đương và chứng minh rằng phạ trù các $(n+1)$ - không gian fuzzy và phạm trù các $(n+1)$ - không gian Chu hoàn toàn đầy đư là đẳng cấu với nhau. Cuối cùng, khi đưa ra các khái niệm về chuẩn, trung bình và độ lệch tiêu chuẩn, chúng tôi chỉ ra rằng các đại lượng này là bất biến trò chơi.
\end{abstract}

\section{INTRODUCTION}

This work is motivated by recent attempt to model information flow in distributed system of Bariwise and Seligman in 1977 as well as the work of V.R. Pratt in computer science in which a general algebraic scheme, known as Chu space, is systematically used. In this paper we continue to study the finite-dimensional Chu space introduced in [3]. This paper is organized as follows. In section we recall the notion of finite-dimensional Chu space in general settings, and define some numerical data which used in section 4 . In section 3 we introduce a new class of covariant functors, called the " $(n+1)$-fuzzy functors", from the $n$-set category into the category of $(n+1)$-fuzzy spaces. We show that the $(n+1)$ - fuzzy category is an equivalent system and prove that the two categories of $(n+1)$ - fuzzy spaces and of fully complete $(n+1)$ - Chu spaces are isomorphic. In section 4 we define some statistical data as norm, mean, standard deviation of a game space. These data are proved to be game invariance.

\section{FINITE-DIMENSIONAL CHU SPACES}

By a $(n+m)-C h u$ space we mean the set $\tilde{C}=\left(X_{1} \times X_{2} \times \ldots \times X_{n} ; f ; A_{1} \times A_{2} \times \ldots \times A_{m}\right)$, where $X_{i}, A_{j}(i=1, \ldots, n ; j=1, \ldots, m)$ are arbitrary sets and $f: X_{1} \times \ldots \times X_{n} \times A_{1} \times \ldots \times A_{m} \rightarrow[0,1]$ is a map, called the probability function of $\tilde{C}$.

If $\tilde{C}=\left(X_{1} \times X_{2} \times \ldots \times X_{n} ; f ; A_{1} \times A_{2} \times \ldots \times A_{m}\right)$ and $\tilde{D}=\left(Y_{1} \times Y_{2} \times \ldots \times Y_{n} ; g ; B_{1} \times B_{2} \times \ldots \times B_{m}\right)$ áre $(n+m)$-Chu spaces, then a $(n+m)-C h u$ morphism $\Phi: \tilde{C} \rightarrow \widetilde{D}$ is a $(n+m)$-tuple of maps $\Phi=\left(\varphi_{1}, \varphi_{2}, \ldots, \varphi_{n} ; \psi_{1}, \psi_{2}, \ldots, \psi_{m}\right)$, with $\varphi_{i}: X_{i} \rightarrow Y_{i}$ for $i=1, \ldots, n$ and $\psi_{j}: B_{j} \rightarrow A_{j}$ for $j=1, \ldots, m$ such that the diagram below commutes:

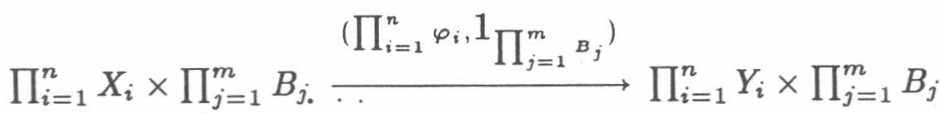

$$
\begin{aligned}
& \left(1_{\prod_{i=1}^{n} x_{i}}, \prod_{j=1}^{m} \psi_{j}\right) \downarrow \downarrow \downarrow g \\
& \prod_{i=1}^{n} X_{i} \times \prod_{j=1}^{m} A_{j} \longrightarrow \quad[0,1]
\end{aligned}
$$

where ${ }^{1} \prod_{i=1}^{n} X_{i}, 1_{\prod_{j=1}^{m} B_{j}}$ denote identity maps. That is 
or equivalently,

$$
f \circ\left(1 \prod_{i=1}^{n} X_{i}, \prod_{j=1}^{m} \psi_{j}\right)=g \circ\left(\prod_{i=1}^{n} \varphi_{i}, 1 \prod_{j=1}^{m} B_{j}\right)
$$

$$
f\left(\prod_{i=1}^{n} x_{i} \times \prod_{j=1}^{m} \psi_{j}\left(b_{j}\right)\right)=g\left(\prod_{i=1}^{n} \varphi_{i}\left(x_{i}\right) \times \prod_{j=1}^{m} b_{j}\right) \text { for } \prod_{i=1}^{n} x_{i} \in \prod_{i=1}^{n} X_{i} \text { and } \prod_{j=1}^{m} b_{j} \in \prod_{j=1}^{m} B_{j} .
$$

If $\Phi=\left(\varphi_{1}, \ldots, \varphi_{n} ; \psi_{1}, \ldots, \psi_{m}\right): \tilde{C}=\left(X_{1} \times \ldots \times X_{n} ; f ; A_{1} \times \ldots \times A_{m}\right) \rightarrow \tilde{D}=\left(Y_{1} \times \ldots \times Y_{n} ; g ; B_{1} \times\right.$ $\left.\ldots \times B_{m}\right)$ is a $(n+m)$ - Chu morphism, then the $(n+m)$ - Chu space $\left(\prod_{i=1}^{n} X_{i} ; f \times_{\Phi} g ; \prod_{j=1}^{m} B_{j}\right)$, where

$$
\left(f \times_{\Phi} g\right)=f \circ\left(1 \prod_{i=1}^{n} X_{i}, \prod_{j=1}^{m} \psi_{j}\right)=g \circ\left(\prod_{i=1}^{n} \varphi_{i}, 1 \prod_{j=1}^{m} B_{j}\right)
$$

is called the cross product of $\widetilde{C}$ and $\widetilde{D}$ over $\Phi$, denoted by $\widetilde{C} \times_{\Phi} \widetilde{D}$.

For $\prod_{i=1}^{n} x_{i} \in \prod_{i=1}^{n} X_{i}$ we define the following notation:

1. The number $\left\|\prod_{i=1}^{n} x_{i}\right\|^{*}=\sup \left\{f\left(\prod_{i=1}^{n} x_{i} \times \prod_{j=1}^{m} a_{j}\right): \prod_{j=1}^{m} a_{j} \in \prod_{j=1}^{m} A_{j}\right\}$ is called the upper value of $\prod_{i=1}^{n} x_{i}$.

2. The number $\left\|\prod_{i=1}^{n} x_{i}\right\|_{*}=\inf \left\{f\left(\prod_{i=1}^{n} x_{i} \times \prod_{j=1}^{m} a_{j}\right): \prod_{j=1}^{m} a_{j} \in \prod_{j=1}^{m} A_{j}\right\}$ is called the lower value of $\prod_{i=1}^{n} x_{i}$.

3. The number $\left\|\prod_{i=1}^{n} x_{i}\right\|=\frac{1}{2}\left(\left\|\prod_{i=1}^{n} x_{i}\right\|^{*}+\left\|\prod_{i=1}^{n} x_{i}\right\|_{*}\right)$ is called the value of $\prod_{i=1}^{n} x_{i}$.

4. The number $d\left(\prod_{i=1}^{n} x_{i}\right)=\left\|\prod_{i=1}^{n} x_{i}\right\|^{*}-\left\|\prod_{i=1}^{n} x_{i}\right\|_{*}$ is called the deviation of $\prod_{i=1}^{n} x_{i}$.

For $(n+m)$ - Chu spaces $\widetilde{C}=\left(X_{1} \times \ldots \times X_{n} ; f ; A_{1} \times \ldots \times A_{m}\right)$ and $\widetilde{D}=\left(Y_{1} \times \ldots \times Y_{n} ; g ; B_{1} \times \ldots \times B_{m}\right)$ let $\mathcal{M}(\widetilde{C}, \widetilde{D})$ denote the set of all $(n+m)$-Chu morphisms from $\widetilde{C}$ into $\widetilde{D}$. If $\mathcal{M}(\widetilde{C}, \widetilde{D}) \neq \emptyset$, then we say that $\widetilde{C}$ is dominated by $\widetilde{D}$ and denote $\widetilde{C} \preceq \widetilde{D}$. We say that $\widetilde{C}$ and $\widetilde{D}$ are equivalent, denoted by $\widetilde{C} \approx \widetilde{D}$, if $\widetilde{C} \preceq \widetilde{D}$ and $\widetilde{D} \preceq \widetilde{C} ; \widetilde{C}$ and $\widetilde{D}$ are connected if either $\widetilde{C} \preceq \widetilde{D}$ or $\widetilde{D} \preceq \widetilde{C}$. A class of $(\mathrm{n}+\mathrm{m})$-Chu spaces $\mathcal{G}$ is called a connected system if any two members of $\mathcal{G}$ are connected. If $\tilde{C} \approx \tilde{D}$ for every $\widetilde{C}, \widetilde{D} \in \mathcal{G}$, then we say that $\mathcal{G}$ is an equivalent system. A connected system is called a closed system if $\mathcal{G}$ is closed under cross products. That is, $\widetilde{C} \times_{\Phi} \widetilde{D} \in \mathcal{G}$ for any $\widetilde{C}, \widetilde{D} \in \mathcal{G}$ and $\Phi \in \mathcal{M}(\widetilde{C}, \widetilde{D})$. A complete system is a closed equivalent system.

Let $\tilde{C}=\left(X_{1} \times \ldots \times X_{n} ; f ; A_{1} \times \ldots \times A_{m}\right)$ and $\tilde{D}=\left(Y_{1} \times \ldots \times Y_{n} ; g ; B_{1} \times \ldots \times B_{m}\right)$ be $(n+m)$ Chu spaces, we say that $\widetilde{C}$ and $\widetilde{D}$ are isomorphic, denoted by $\widetilde{C} \cong \widetilde{D}$, if $\widetilde{C}$ and $\widetilde{D}$ are isomorphic objects in the category $C$ of $(n+m)$ - Chu spaces. It is easy to see that a $(n+m)-$ Chu morphism $\Phi=\left(\varphi_{1}, \ldots, \varphi_{n} ; \psi_{1}, \ldots, \psi_{m}\right):\left(X_{1} \times \ldots \times X_{n} ; f ; A_{1} \times \ldots \times A_{m}\right) \rightarrow\left(Y_{1} \times \ldots \times Y_{n} ; g ; B_{1} \times \ldots \times B_{m}\right)$ is an isomorphism if and only if $\varphi_{i}: X_{i} \rightarrow Y_{i}$ for $i=1, \ldots, n$ and $\psi_{j}: B_{j} \rightarrow A_{j}$ for $j=1, \ldots, m$ are one-to-one and onto.

If $\Phi=\left(\varphi_{1}, \ldots, \varphi_{n} ; \psi_{1}, \ldots, \psi_{m}\right)$ is a $(n+m)$ - monomorphism, then we say that $\widetilde{C}=\left(X_{1} \times \ldots \times\right.$ $\left.X_{n} ; f ; A_{1} \times \ldots \times A_{m}\right)$ is a subspace of $\widetilde{D}=\left(Y_{1} \times \ldots \times Y_{n} ; g ; B_{1} \times \ldots \times B_{m}\right)$, denoted by $\widetilde{C} \subseteq \tilde{D}$. It is easy to see that a $(n+m)$-Chu morphism $\Phi=\left(\varphi_{1}, \ldots, \varphi_{n} ; \psi_{1}, \ldots, \psi_{m}\right):\left(X_{1} \times \ldots \times X_{n} ; f ; A_{1} \times \ldots \times A_{m}\right) \rightarrow$ $\left(Y_{1} \times \ldots \times Y_{n} ; g ; B_{1} \times \ldots \times B_{m}\right)$ is a mornomorphism iff $\varphi_{i}: X_{i} \rightarrow Y_{i}$ for $i=1, \ldots, n$ are one-to-one and $\psi_{j}: B_{j} \rightarrow A_{j}$ for $j=1, \ldots, m$ are onto.

\section{FUZZY SPACE AND FUZZY FUNCTOR}

Recall that by a fuzzy subset of a set $X=\prod_{i=1}^{n} X_{i}$, we mean a fuction $f: X \rightarrow[0,1]$, see [3]. Observe that if $A$ is a subset of $X$, then the characteristic function $\chi_{A}$ of $A$ is a fuzzy subset of $X$. So by identifying $A$ with $\chi_{A}$ we can say that any subset of $X$ is a fuzzy subset of $X$. A fuzzy subset of $X$ is also simply called a fuzzy set.

Let $S$ denote the category of sets. For a given set $X=\prod_{i=1}^{n} X_{i}$, let $X^{*}=[0,1]^{X}$ denote collection of all fuzzy sets of $X$. 
For any map $\alpha: X=X_{1} \times X_{2} \times \ldots \times X_{n} \rightarrow Y=Y_{1} \times Y_{2} \times \ldots \times Y_{n}$ we define the conjugate $\alpha^{*}: Y^{*} \rightarrow X^{*}$ of $\alpha$ by the formula

for every $x \in X$ and $a \in Y^{*}$.

$$
\alpha^{*}(a)(x)=a(\alpha(x))
$$

It is easy to see that

$$
(\beta \alpha)^{*}=\alpha^{*} \beta^{*} \text { for every } \alpha: X \rightarrow Y \text { and } \beta: Y \rightarrow Z .
$$

For any set $A \subset X^{*}$ we define $f_{A}: X_{1} \times X_{2} \times \ldots \times X_{n} \times A \rightarrow[0,1]$ by

$$
f_{A}\left(x_{1}, \ldots, x_{n}, a\right)=a\left(x_{1}, \ldots, x_{n}\right) \text { for }\left(x_{1}, \ldots, x_{n}, a\right) \in X_{1} \times X_{2} \times \ldots \times X_{n} \times A .
$$

Clearly that $\tilde{C}=\left(X_{1} \times X_{2} \times \ldots \times X_{n} ; f_{A} ; A\right)$ is a $(n+1)$ - Chu space. This space is called a $(n+1)$ pre-fuzzy space on $X=X_{1} \times X_{2} \times \ldots \times X_{n}$. In the case $A=\left(X_{1} \times X_{2} \times \ldots \times X_{n}\right)^{*}$, the $(n+1)$ - Chu space $F(X)=\left(X_{1} \times X_{2} \times \ldots \times X_{n} ; f_{X^{*}} ; X^{*}\right)$ is uniquely determined by $X=X_{1} \times X_{2} \times \ldots \times X_{n}$, and is called $(n+1)$ - fuzzy space associated with $X$, or shortly a $(n+1)$-fuzzy space.

The category of $(n+1)$--pre-fuzzy spaces with $(n+1)$ - Chu morphisms is called the $(n+1)$ - prefuzzy category, denoted by $\mathcal{F}_{P}$. The $(n+1)$ - fuzzy category, denoted by $\mathcal{F}$, is the subcategory of $\mathcal{F}_{P}$ consisting of fuzzy spaces.

Observe that a $(n+1)$ - Chu morphism $\Phi: \tilde{C}=\left(X_{1} \times X_{2} \times \ldots \times X_{n} ; f_{A} ; A\right) \rightarrow \tilde{D}=\left(Y_{1} \times Y_{2} \times\right.$ $\left.\ldots \times Y_{n} ; f_{B} ; B\right)$ in the $(n+1)$ - pre-fuzzy category is a collection of maps $\Phi=\left(\varphi_{1}, \varphi_{2}, \ldots, \varphi_{n} ; \psi\right)$, where

$$
\prod_{i=1}^{n} \varphi_{i}: \prod_{i=1}^{n} X_{i} \rightarrow \prod_{i=1}^{n} Y_{i} \text { with }\left(\prod_{i=1}^{n} \varphi_{i}\right)\left(\prod_{i=1}^{n} x_{i}\right)=\prod_{i=1}^{n} \varphi_{i}\left(x_{i}\right) \in \prod_{i=1}^{n} Y_{i}
$$

and $\psi: B \rightarrow A$ satisfy the condition

$$
\psi(b)\left(\prod_{i=1}^{n} x_{i}\right)=b\left(\prod_{i=1}^{n} \varphi_{i}\left(x_{i}\right)\right) \text { for }\left(x_{1}, \ldots, x_{n}, b\right) \in X \times B .
$$

It is easy to see that, in general $(n+1)$ - Chu spaces are not connected. Forturnately it is not the case in the $(n+1)$ - fuzzy category. In fact, we have the following theorem.

Theorem 1. The $(n+1)$ - fuzzy category $₹$ is an equivalent system.

Proof. Let $X=X_{1} \times X_{2} \times \ldots \times X_{n}, Y=Y_{1} \times Y_{2} \times \ldots \times Y_{n}$, we need to show that $\mathcal{M}(F(X), F(Y)) \neq \emptyset$ for any $(n+1)$ - fuzzy spaces $F(X)=\left(X_{1} \times X_{2} \times \ldots \times X_{n} ; f_{X^{*}} ; X^{*}\right)$ and $F(Y)=\left(Y_{1} \times Y_{2} \times \ldots \times Y_{n} ; f_{Y^{*}} ; Y^{*}\right)$.

Let $\alpha: X \rightarrow Y$ be any map (in the set category). Define $\alpha^{*}: Y^{*} \rightarrow X^{*}$ by $\alpha^{*}\left(y^{*}\right)\left(x_{1}, \ldots, x_{n}\right)=$ $y^{*}\left(\alpha\left(x_{1}, \ldots, x_{n}\right)\right)$ for $\left(x_{1}, \ldots, x_{n}\right) \in X_{1} \times X_{2} \times \ldots \times X_{n}$ and $y^{*} \in Y^{*}$.

We have

$$
\begin{aligned}
\alpha^{*}\left(y^{*}\right)\left(x_{1}, \ldots, x_{n}\right) & =f_{X^{*}}\left(x_{1}, \ldots, x_{n}, \alpha^{*}\left(y^{*}\right)\right) \\
& =y^{*}\left(\alpha\left(x_{1}, \ldots, x_{n}\right)\right) \\
& =f_{Y^{*}}\left(\alpha\left(x_{1}, \ldots, x_{n}\right), y^{*}\right) .
\end{aligned}
$$

Therefore the diagram bellow commutes

$$
\begin{array}{rc}
\prod_{i=1}^{n} X_{i} \times Y^{*} \stackrel{\left(\alpha, 1_{Y^{*}}\right)}{\longrightarrow} & \prod_{i=1}^{n} Y_{i} \times Y^{*} \\
\left.{ }^{(1} \prod_{i=1}^{n} x_{i}{ }^{*}\right) \downarrow & \downarrow_{Y^{*}} \\
\prod_{i=1}^{n} X_{i} \times X^{*} \stackrel{ }{\longrightarrow} \underset{f_{X^{*}}}{\longrightarrow} & {[0,1] .}
\end{array}
$$

Thus, $\Phi=\left(\alpha, \alpha^{*}\right) \in \mathcal{M}(F(X), F(Y))$ and the theorem is proved.

By $n$-set we mean the cartesian product $X=X_{1} \times \ldots \times X_{n}$. We will show that $F(X)=$ $\left(X_{1} \times \ldots \times X_{n} ; f_{X^{*}} ; X^{*}\right)$ is a covariant functor from the n-set category $S$ into the $(n+1)$-fuzzy category $₹$ and then $\mathrm{F}$ will be called a $(n+1)$ - fuzzy functor. 
In fact, let $\alpha: \prod_{i=1}^{n} X_{i} \rightarrow \prod_{i=1}^{n} Y_{i}$ be a map. Define $F(\alpha): F(X) \rightarrow F(Y)$ by $F(\alpha)=\left(\alpha, \alpha^{*}\right)$, where $\alpha^{*}: Y^{*} \rightarrow X^{*}$ is the conjugate of $\alpha$.

We observe that

$$
F(\beta \alpha)=\left(\beta \alpha,(\beta \alpha)^{*}\right)=\left(\beta \alpha, \alpha^{*} \beta^{*}\right)=F(\beta) F(\alpha)
$$

for any $\alpha: \prod_{i=1}^{n} X_{i} \rightarrow \prod_{i=1}^{n} Y_{i}$ and $\beta: \prod_{i=1}^{n} Y_{i} \rightarrow \prod_{i=1}^{n} Z_{i}$. Therefore $F$ preserves the composition.

Theorem 2. The two categories $\mathcal{F}$ and $C_{F}$ are isomophic.

Proof. The functor $F$ defined in the proof of Theorem 2 in [3] is an isomorphism between the $(n+1)$ fuzzy category $\mathcal{F}$ and the category $C_{F}$ of fully complete $(n+1)$ - Chu spaces.

From Theorem 1 and Theorem 2 we get:

Corollary 1. The category $C_{F}$ of all fully complete $(n+1)-$ Chu spaces is an equivalent system.

Remark 1. Since any subset of a set $X$ is a fuzzy set, we can consider the family $A=2^{X} \subset$ $X^{*}$ consisting of all subsets of $X=X_{1} \times \ldots \times X_{n}$. The resulting $(n+1)$ - pre-fuzzy space $D(X)=$ $\left(\prod_{i=1}^{n} X_{i} ; f_{2} x ; 2^{X}\right)$ will be called the $(n+1)$ - Crisp space associated with $X$, and the category $D$ of all crisp spaces is called the crisp category.

We will show that

Proposition 1. Every $(n+1)$ - Crisp space is biextensional.

Proof. By Proposition 7 in [3], every $(n+1)$ - pre-fuzzy space is separated, therefore we need to claim that it is extensional.

Assume

$$
0=\left\|\prod_{i=1}^{n} x_{i}-\prod_{i=1}^{n} y_{i}\right\|=\sup \left\{\left|f\left(\prod_{i=1}^{n} x_{i}, a\right)-f\left(\prod_{i=1}^{n} y_{i}, a\right)\right|: a \in 2^{X}\right\}
$$

then $a\left(\prod_{i=1}^{n} x_{i}\right)=a\left(\prod_{i=1}^{n} y_{i}\right)$ for every $a \in 2^{X}$. From that it follows $\prod_{i=1}^{n} x_{i}=\prod_{i=1}^{n} y_{i}$, since if it is not the case, setting $a=\chi_{\left\{\prod_{i=1}^{n} x_{i}\right\}} \in 2^{X}$, we get $a\left(\prod_{i=1}^{n} x_{i}\right)=1$, but $a\left(\prod_{i=1}^{n} y_{i}\right)=0$.

The crisp category $D$ is a subcategory of $\mathcal{F}$. We observe that

Proposition 2. The map $D$ defined in Remark 1 is a covariant functor from the $n$-set category $S$ into the $(n+1)$ - crisp category $D$.

Proof. Let $\alpha: \prod_{i=1}^{n} X_{i} \rightarrow \prod_{i=1}^{n} Y_{i}$ be a map. Then the morphism

$$
D(\alpha): D(X)=\left(\prod_{i=1}^{n} X_{i} ; f_{2} x ; 2^{X}\right) \rightarrow D(Y)=\left(\prod_{i=1}^{n} Y_{i} ; f_{2^{Y}} ; 2^{Y}\right)
$$

is defined by $D(\alpha)=\left(\alpha, \alpha^{-1}\right)$, where $\alpha^{-1}(D) \in 2^{X}$ for every $D \in 2^{Y}$.

We will show that the following diagram commutes

$$
\begin{array}{rc}
\prod_{i=1}^{n} X_{i} \times 2^{Y} & \stackrel{\left(\alpha, 1_{2} Y\right)}{\longrightarrow} \prod_{i=1}^{n} Y_{i} \times 2^{Y} \\
\left.{ }^{(1} \prod_{i=1}^{n} x_{i}{ }^{, \alpha^{-1}}\right) & \\
\prod_{i=1}^{n} X_{i} \times 2^{X} \stackrel{f_{2} Y}{\underset{f_{2} X}{\longrightarrow}} & {[0,1] .}
\end{array}
$$

In fact, by definition of $f_{2} x$ and $f_{2^{Y}}$, we need to claim that

$$
\alpha^{-1}(b)\left(\prod_{i=1}^{n} x_{i}\right)=b\left(\alpha\left(\prod_{i=1}^{n} x_{i}\right)\right) \text { for every } b \in 2^{Y} .
$$


Since $\alpha^{-1}(b)$ and $b$ are two characteristic functions of the set $\alpha^{-1}(b)$ in the space $2^{X}$ and $2^{Y}$, respectively, they admit only two values 0 or 1 . If $\alpha^{-1}(b)\left(\prod_{i=1}^{n} x_{i}\right)=1$, then $\prod_{i=1}^{n} x_{i} \in \alpha^{-1}(b)$ which implies $\alpha\left(\prod_{i=1}^{n} x_{i}\right) \in b$, hence $b(\alpha x)=1$. If $\alpha^{-1}(b)\left(\prod_{i=1}^{n} x_{i}\right)=0$, then $\prod_{i=1}^{n} x_{i} \notin \alpha^{-1}(b)$ which implies $\alpha\left(\prod_{i=1}^{n} x_{i}\right) \notin b$, hence $b\left(\alpha\left(\prod_{i=1}^{n} x_{i}\right)\right)=0$.

Thus, in both cases we have

$$
\alpha^{-1}(b)\left(\prod_{i=1}^{n} x_{i}\right)=b\left(\alpha\left(\prod_{i=1}^{n} x_{i}\right)\right) \text { for } \prod_{i=1}^{n} x_{i} \in \prod_{i=1}^{n} X_{i} .
$$

Therefore the proposition is proved.

\section{GAME SPACE AND THE GAME INVARIANCE THEOREM}

Given a set $A=\prod_{j=1}^{m} A_{j}$, by a game space over $\mathrm{A}=\prod_{j=1}^{m} A_{j}$, we mean a $(n+m)$ - Chu space $\tilde{G}=\left(\prod_{i=1}^{n} X_{i} ; f ; \prod_{j=1}^{m} A_{j}\right)$, where:

1. $\prod_{i=1}^{n} X_{i}$ is a cartesian product of finite sets, called the team game. If $\prod_{i=1}^{n} x_{i} \in \prod_{i=1}^{n} X_{i}$, then $\prod_{i=1}^{n} x_{i}$ is called the players of the game space $\widetilde{G}$.

2. $\prod_{j=1}^{m} A_{j}$ is a cartesian product of any sets, called the field game. If $\prod_{j=1}^{m} a_{j} \in \prod_{j=1}^{m} A_{j}$, then $\prod_{j=1}^{m} a_{j}$ is called a position in the field game $\prod_{j=1}^{m} A_{j}$.

3. $f\left(\prod_{i=1}^{n} x_{i}, \prod_{j=1}^{m} a_{j}\right)$ is called the winning probability of the players $\prod_{i=1}^{n} x_{i}$ while they are in the position $\prod_{j=1}^{m} a_{j}$ in the field game.

Observe that if $\tilde{G}=\left(\prod_{i=1}^{n} X_{i} ; f ; \prod_{j=1}^{m} A_{j}\right)$ is a game space, then the upper value $\left\|\prod_{i=1}^{n} x_{i}\right\|^{*}$ measures the 11skill" of $\prod_{i=1}^{n} x_{i}$ in the best situation and the lower value $\left\|\prod_{i=1}^{n} x_{i}\right\|_{*}$ measures the "skill" of the set $\prod_{i=1}^{n} x_{i}$ in the worst situation.

Dually, for a state $\prod_{j=1}^{m} a_{j} \in \prod_{j=1}^{m} A_{j}$ the upper value $\left\|\prod_{j=1}^{m} a_{j}\right\|^{*}$ describes the quality of the position $\prod_{j=1}^{m} a_{j}$ in hands of the best players and the lower value $\left\|\prod_{j=1}^{m} a_{j}\right\|_{*}$ describes the quality of the position $\prod_{j=1}^{m} a_{j}$ if the worst players are staying there.

Since the set $\prod_{i=1}^{n} X_{i}$ of a game space $\tilde{G}=\left(\prod_{i=1}^{n} X_{i} ; f ; \prod_{j=1}^{m} A_{j}\right)$ is finite, we can define the following statistical data for a game space:

1. The number $\|\tilde{G}\|=\sqrt{\sum_{\prod_{i=1}^{n} x_{i} \in \prod_{i=1}^{n} x_{i}}\left\|\prod_{i=1}^{n} x_{i}\right\|^{2}}$ is called the norm of $\tilde{G}$.

2. The number $D(\tilde{G})=\sqrt{\sum_{\prod_{i=1}^{n} x_{i} \in \prod_{i=1}^{n} X_{i}}\left[d\left(\prod_{i=1}^{n} x_{i}\right)\right]^{2}}$ is called the standard deviation of $\tilde{G}$.

3. The number $M(\tilde{G})=\frac{1}{\left|\prod_{i=1}^{n} X_{i}\right|} \sum_{\prod_{i=1}^{n} x_{i} \in \prod_{i=1}^{n} X_{i}}\left\|\prod_{i=1}^{n} x_{i}\right\|$, where $\left|\prod_{i=1}^{n} X_{i}\right|$ denotes the cardinality of $\prod_{i=1}^{n} X_{i}$, is called the mean of $\tilde{G}$. follows:

Now given a set $\prod_{j=1}^{m} A_{j}$, we define the game category over the field $\prod_{j=1}^{m} A_{j}$, denoted $\mathcal{G}_{A}$ as

1. The objects of $\mathcal{g}_{A}$ are game spaces over $\prod_{j=1}^{m} A_{j}$.

2. If $\widetilde{S}=\left(\prod_{i=1}^{n} X_{i} ; f ; \prod_{j=1}^{m} A_{j}\right)$ and $\tilde{T}=\left(\prod_{i=1}^{n} Y_{i} ; g ; \prod_{j=1}^{m} A_{j}\right)$ are two game spaces over $\prod_{j=1}^{m} A_{j}$, then a morphism $\Phi=\left(\varphi_{1}, \ldots, \varphi_{n} ; \prod_{\prod_{j=1}^{m} A_{j}}\right): \widetilde{S} \rightarrow \widetilde{T}$, where $\varphi_{i}: X_{i} \rightarrow Y_{i}$, for $\mathrm{i}=1, \ldots, \mathrm{n}$ are maps satisfying the condition:

$$
f\left(\prod_{i=1}^{n} x_{i} \times \prod_{j=1}^{m} a_{j}\right) \leq g\left(\prod_{i=1}^{n} \varphi_{i}\left(x_{i}\right) \times \prod_{j=1}^{m} a_{j}\right)
$$

for $\prod_{i=1}^{n} x_{i} \in \prod_{i=1}^{n} X_{i}$ and $\prod_{j=1}^{m} a_{j} \in \prod_{j=1}^{m} A_{j}$.

Consequently morphisms in the game category $\mathcal{G}_{A}$ are $(n+m)$-Chu upper-morphisms. 
The existence of a $(n+m)$ - morphism $\Phi: \widetilde{S} \rightarrow \tilde{T}$ in the game category over the field $\prod_{j=1}^{m} A_{j}$ implies that for any set of players $\prod_{i=1}^{n} x_{i}$ of the team $\prod_{i=1}^{n} X_{i}$, there exists a set of players $\prod_{i=1}^{n} \varphi_{i}\left(x_{i}\right)$ of the team $\prod_{i=1}^{n} Y_{i}$ such that at any situation $\prod_{j=1}^{m} a_{j}$ in the game field $\prod_{j=1}^{m} A_{j}$, the set of players $\prod_{i=1}^{n} \varphi_{i}\left(x_{i}\right)$ have better chance to win than the set of players $\prod_{i=1}^{n} x_{i}$ at the same situations $\prod_{j=1}^{m} a_{j}$. It follows that the team $\prod_{i=1}^{n} Y_{i}$ have some advantages over the team $\prod_{i=1}^{n} X_{i}$ in the field $\prod_{j=1}^{m} A_{j}$.

We have

Lemma 1. If $\left.\widetilde{S}=\left(\prod_{i=1}^{n} X_{i}\right) ; f ; \prod_{j=1}^{m} A_{j}\right)$ is a subset of $\left.\widetilde{G}=\left(\prod_{i=1}^{n} Y_{i}\right) ; g ; \prod_{j=1}^{m} A_{j}\right)$, then $\|\widetilde{S}\| \leq$ $\|\tilde{G}\|$.

Proof. Since the game space $\left.\widetilde{S}=\left(\prod_{i=1}^{n} X_{i}\right) ; f ; \prod_{j=1}^{m} A_{j}\right)$ is a subset of the the game space $\tilde{G}=$ $\left.\left(\prod_{i=1}^{n} Y_{i}\right) ; g ; \prod_{j=1}^{m} A_{j}\right)$, there is a monorphism $\Phi=\left(\varphi_{1}, \ldots, \varphi_{n} ; \psi_{1}, \ldots, \psi_{m}\right): \widetilde{S} \rightarrow \widetilde{G}$ with $\varphi_{i}: X_{i} \rightarrow$ $Y_{i}$ for $i=1, \ldots, n$ are one-to-one and $\psi_{j}: A_{j} \rightarrow A_{j}$ are identical maps for $j=1, \ldots, m$, so that

$$
f\left(\prod_{i=1}^{n} x_{i} \times \prod_{j=1}^{m} a_{j}\right) \leq g\left(\prod_{i=1}^{n} \varphi_{i}\left(x_{i}\right) \times \prod_{j=1}^{m} a_{j}\right)
$$

for $\prod_{i=1}^{n} x_{i} \in \prod_{i=1}^{n} X_{i}$ and $\prod_{j=1}^{m} a_{j} \in \prod_{j=1}^{m} A_{j}$. We have

$$
\begin{aligned}
\left\|\prod_{i=1}^{n} x_{i}\right\|^{*} & =\sup \left\{f\left(\prod_{i=1}^{n} x_{i} \times \prod_{j=1}^{m} a_{j}\right): \prod_{j=1}^{m} a_{j} \in \prod_{j=1}^{m} A_{j}\right\} \\
& \leq \sup \left\{g\left(\prod_{i=1}^{n} \varphi_{i}\left(x_{i}\right) \times \prod_{j=1}^{m} a_{j}\right): \prod_{j=1}^{m} a_{j} \in \prod_{j=1}^{m} A_{j}\right\} \\
& =\sup \left\{g\left(\prod_{i=1}^{n} y_{i} \times \prod_{j=1}^{m} a_{j}\right): \prod_{j=1}^{m} a_{j} \in \prod_{j=1}^{m} A_{j}\right\} \\
& =\left\|\prod_{i=1}^{n} y_{i}\right\|^{*},
\end{aligned}
$$

and

$$
\begin{aligned}
\left\|\prod_{i=1}^{n} x_{i}\right\|_{*} & =\inf \left\{f\left(\prod_{i=1}^{n} x_{i} \times \prod_{j=1}^{m} a_{j}\right): \prod_{j=1}^{m} a_{j} \in \prod_{j=1}^{m} A_{j}\right\} \\
& \leq \inf \left\{g\left(\prod_{i=1}^{n} \varphi_{i}\left(x_{i}\right) \times \prod_{j=1}^{m} a_{i}\right): \prod_{j=1}^{m} a_{j} \in \prod_{j=1}^{m} A_{j}\right\} \\
& =\inf \left\{g\left(\prod_{i=1}^{n} y_{i} \times \prod_{j=1}^{m} a_{i}\right): \prod_{j=1}^{m} a_{j} \in \prod_{j=1}^{m} A_{j}\right\} \\
& =\left\|\prod_{i=1}^{n} y_{i}\right\|_{*} .
\end{aligned}
$$

So

$$
\left\|\prod_{i=1}^{n} x_{i}\right\| \leq\left\|\prod_{i=1}^{n} y_{i}\right\| .
$$

On the other hand, since $\varphi_{i}$ are one-to-one for $i=1, \ldots, n,\left|\prod_{i=1}^{n} X_{i}\right| \leq\left|\prod_{i=1}^{n} Y_{i}\right|$.

Therefore

$$
\sqrt{\sum_{\prod_{i=1}^{n}}\left\|\prod_{x_{i} \in \prod_{i=1}^{n} x_{i}}^{n} x_{i}\right\|^{2}} \leq \sqrt{\sum_{\prod_{i=1}^{n}} \sum_{y_{i} \in \prod_{i=1}^{n} Y_{i}}\left\|\prod_{i=1}^{n} y_{i}\right\|^{2}}
$$


Consequently

$$
\|\widetilde{S}\| \leq\|\widetilde{G}\|
$$

Remark 2. With the same assumption in the Lemma 1, we will show that $M(\tilde{S}) \leq M(\tilde{G})$ is in general not true.

In fact, suppose that for a given set $\prod_{i=1}^{n} X_{i}$, let $\prod_{i=1}^{n} Y_{i}=\prod_{i=1}^{n} X_{i} \cup\left\{\prod_{i=1}^{n} x_{i}^{0}\right\}$, where $\prod_{i=1}^{n} x_{i}^{0} \notin \prod_{i=1}^{n} X_{i}$. We put

$$
g\left(\prod_{i=1}^{n} y_{i} \times \prod_{j=1}^{m} a_{j}\right)=f\left(\prod_{i=1}^{n} x_{i} \times \prod_{j=1}^{m} a_{j}\right) \text { if } \prod_{i=1}^{n} y_{i}=\prod_{i=1}^{n} x_{i}
$$

and

$$
g\left(\prod_{i=1}^{n} x_{i}^{0} \times \prod_{j=1}^{m} a_{j}\right)=0 \text { for every } \prod_{j=1}^{m} a_{j} \in \prod_{j=1}^{m} A_{j} .
$$

Then $\left\|\prod_{i=1}^{n} x_{i}^{0}\right\|=0$ and $\tilde{S}=\left(\prod_{i=1}^{n} X_{i} ; f ; \prod_{j=1}^{m} A_{j}\right)$ is a subset of the $\tilde{G}=\left(\prod_{i=1}^{n} Y_{i} ; g ; \prod_{j=1}^{m} A_{j}\right)$.

Let $\Phi=\left(\varphi_{1}, \ldots, \varphi_{n}, 1 \prod_{j=1}^{m} A_{j}\right): \tilde{S} \rightarrow \tilde{G}$, be a morphism from $\tilde{S}$ into $\tilde{G}$. Then

$$
f\left(\prod_{i=1}^{n} x_{i} \times \prod_{j=1}^{m} a_{j}\right)=g\left(\prod_{i=1}^{n} \varphi_{i}\left(x_{i}\right) \times \prod_{j=1}^{m} a_{j}\right) \text { for every } \prod_{i=1}^{n} x_{i} \in \prod_{i=1}^{n} X_{i} .
$$

We have

$$
\left\|\prod_{i=1}^{n} x_{i}\right\|=\left\|\prod_{i=1}^{n} \varphi_{i}\left(x_{i}\right)\right\|=\left\|\prod_{i=1}^{n} y_{i}\right\| \text { and }\left|\prod_{i=1}^{n} X_{i}\right|<\left|\prod_{i=1}^{n} Y_{i}\right| .
$$

Hence

$$
\begin{aligned}
M(\tilde{S}) & =\frac{1}{\left|\prod_{i=1}^{n} X_{i}\right|} \sum_{\prod_{i=1}^{n} x_{i} \in \prod_{i=1}^{n} X_{i}}\left\|\prod_{i=1}^{n} x_{i}\right\| \\
& =\frac{1}{\left|\prod_{i=1}^{n} X_{i}\right|}\left(\sum_{\prod_{i=1}^{n}}\left\|\prod_{i} \in \prod_{i=1}^{n} X_{i} x_{i}\right\|+\left\|\prod_{i=1}^{n} x_{i}^{0}\right\|\right) \\
& =\frac{1}{\left|\prod_{i=1}^{n} X_{i}\right|} \sum_{\prod_{i=1}^{n} y_{i} \in \prod_{i=1}^{n} Y_{i}}\left\|\prod_{i=1}^{n} y_{i}\right\| \\
& >\frac{1}{\left|\prod_{i=1}^{n} Y_{i}\right|} \sum_{\prod_{i=1}^{n} y_{i} \in \prod_{i=1}^{n} Y_{i}}\left\|\prod_{i=1}^{n} y_{i}\right\| \\
& =M(\tilde{G}) .
\end{aligned}
$$

It shows that, in this case, $\tilde{S}$ is a subset of $\tilde{G}$ but $M(\tilde{S})>M(\tilde{G})$.

Theorem 3 (The game invariance theorem). The numbers $\|\tilde{G}\|, M(\tilde{G})$ and $D(\tilde{G})$ are invariance in the game category over the field $A$. That is, if $\tilde{S}$ and $\tilde{G}$ are isomorphic, then $\|\tilde{S}\|=\|\tilde{G}\|, M(\tilde{S})=$ $M(\widetilde{G})$ and $D(\widetilde{S})=D(\widetilde{G})$.

Proof. From Lemma 1 it follows $\|\tilde{S}\|=\|\tilde{G}\|$. For every $\prod_{i=1}^{n} x_{i} \in \prod_{i=1}^{n} X_{i}$, since $\tilde{S}$ and $\tilde{G}$ are isomorphic, there exists unique $\prod_{i=1}^{n} y_{i}=\prod_{i=1}^{n} \varphi_{i}\left(x_{i}\right) \in \prod_{i=1}^{n} Y_{i}$, such that $f\left(\prod_{i=1}^{n} x_{i} \times \prod_{j=1}^{m} a_{j}\right)=$ $g\left(\prod_{i=1}^{n} \varphi_{i}\left(x_{i}\right) \times \prod_{j=1}^{m} a_{j}\right)=g\left(\prod_{i=1}^{n} y_{i} \times \prod_{j=1}^{m} a_{j}\right)$. 
We have

$$
\left\|\prod_{i=1}^{n} x_{i}\right\|^{*}=\left\|\prod_{i=1}^{n} \varphi_{i}\left(x_{i}\right)\right\|^{*}=\left\|\prod_{i=1}^{n} y_{i}\right\|^{*} \text { and }\left\|\prod_{i=1}^{n} x_{i}\right\|_{*}=\left\|\prod_{i=1}^{n} \varphi_{i}\left(x_{i}\right)\right\|_{*}=\left\|\prod_{i=1}^{n} y_{i}\right\|_{*} .
$$

It implies that $\left\|\prod_{i=1}^{n} x_{i}\right\|=\left\|\prod_{i=1}^{n} \varphi_{i}\left(x_{i}\right)\right\|=\left\|\prod_{i=1}^{n} y_{i}\right\|$.

Thus

$$
\begin{aligned}
M(\tilde{S}) & =\frac{1}{\left|\prod_{i=1}^{n} X_{i}\right|} \sum_{\prod_{i=1}^{n} x_{i} \in \prod_{i=1}^{n} X_{i}}\left\|\prod_{i=1}^{n} x_{i}\right\| \\
& =\frac{1}{\left|\prod_{i=1}^{n} X_{i}\right|}\left(\sum_{\prod_{i=1}^{n} x_{i} \in \prod_{i=1}^{n} X_{i}}\left\|\prod_{i=1}^{n} \varphi_{i}\left(x_{i}\right)\right\|\right) \\
& =\frac{1}{\prod_{i=1}^{n} Y_{i} \mid}\left(\sum_{\prod_{i=1}^{n} y_{i} \in \prod_{i=1}^{n} Y_{i}}\left\|\prod_{i=1}^{n} y_{i}\right\|\right) \\
& =M(\tilde{G}) .
\end{aligned}
$$

The similar argument proves the equality $D(\tilde{\tilde{S}})=D(\tilde{G})$.

The theorem is proved.

Acknowledgement. The authors are grateful to Prof. N. T. Hung for his helpful suggestion.

\section{REFERENCES}

[1] Barry Mitchell, Theory of Categories, New York and London, 1965.

[2] Nguyen Nhuy and Pham Quang Trinh, Chu spaces, Fuzzy sets and Game Invariances, accepted for publication in Viet. J. Math. (2000).

[3] Nguyen Nhuy, Pham Quang Trinh, and Vu Thi Hong Thanh, Finite dimensional Chu space, Journal of Computer Science and Cybernetics 15 (4) (1999) 7-18.

[4] H. T. Nguyen and E. Walker, A First Course in Fuzzy Logic, Boca Raton, FL: CRC, 1997 (2nd ed., 1999).

[5] V.R. Pratt, Type as processes, via Chu spaces, Electronic Notes in Theoretical Computer Science 7 (1997).

[6] V.R. Pratt, Chu spaces as a sematic bridge between linear logic and mathematics, Electronic Notes in Theoretical Computer Science 12 (1998).

Received October 8, 1999

Revised February 14, 2000

Faculty of Information Technology, Vinh University, Nghe An. 